NASA Technical Memorandum 83704

\title{
A Semi-Direct Procedure Using a Local Relaxation Factor and Its Application to an Internal Flow Problem
}

Sin-Chung Chang

Lewis Research Center

Cleveland, Ohio

Prepared for the

Ninth International Conference on Numerical Methods in Fluid Dynamics cosponsored by CEA, CISI, DRET, CNES, EDF, Framatome, and Novatome Saclay, France, June 25-29, 1984 


\section{A SEMI-DIRECT PROCEDURE USING A LOCAL RELAXATION FACTOR AND ITS APPLICATION TO AN INTERNAL FLOW PROBLEM \\ Sin-Chung Chang \\ NASA Lewis Research Center Cleveland, Ohio, 44135. U.S.A.}

\section{INTRODUCTION}

Generally, fast direct solvers (FDS) ${ }^{1}$ are not directly applicable to a nonseparable elliptic partial differential equation (PDE). This limitation, however, can be circumvented by a semi-direct procedure, i.e., an iterative procedure using FDS algorithms. In this paper, we present an efficient semi-direct procedure which is easy to implement and applicable to a variety of boundary conditions. The current procedure also possesses other highly desirable properties, i.e., (1) the convergence rate does not decrease with an increase of grid cell aspect ratio, and (2) the convergence rate can be estimated using the coefficients of the PDE being solved.

Many elliptic PDE encountered in flow problems can be expressed as

$$
\mathrm{Lu}=\mathrm{h}
$$

where $L$ is a nonseparable second-order linear elliptic operator, $u$ the dependent. variable, and $h$ a given source term. Equation (1.1) may be solved with the iterative procedure:

$$
\nabla^{2}\left(u^{n+1}-u^{n}\right)=-\tau\left(L u^{n}-h\right) \quad:(n=0,1 ; 2, \ldots)
$$

Here $\tau$ is a nonzero relaxation factor and $\nabla^{2}$ the Laplacian which can be directly. inverted by a FOS. In the previous works ${ }^{2-4}$ using Eq..(1.2), $\tau$ is treated as. a constant and the iteration is accelerated by an optimal choice of $\tau$. In this:study; we consider $\tau$ as a spatially varying parameter. The local value of $T$ is chosen to optimize the local convergence rate which is evaluated using a von Neumann analysis.

\section{STABILITY ANALYSIS}

As an initial step, it is assumed that $\tau$ is a constant and

$$
L=a \frac{\partial^{2}}{\partial x^{2}}+2 b \frac{\partial^{2}}{\partial x \partial y}+c \frac{\partial^{2}}{\partial y^{2}} \quad \therefore\left(a>0, c>0, \text { and } a c-b^{2}>0\right)
$$

where $a, b$, and $c$ are constants. Using a uniform grid with grid intervals $\Delta x$ and $\Delta y$, the central difference form of Eq. (1.2) at a grid point $(i, j)$ is

$$
\ddot{p}\left(u_{i, j}^{n+1}\right)-\dot{p}\left(u_{i, j}^{n}\right)=-\tau\left(\ddot{L}\left(u_{i, j}^{n}\right)-h_{i j}\right)
$$

Here $h_{i j}$ is the source term and the operators $\vec{p}$ and $\ddot{L}$, respectively, are defined by

$$
\begin{aligned}
& \ddot{p}\left(u_{i, j}^{n}\right)=(\Delta x)^{-2}\left(u_{i+1, j}^{n}+u_{i-1, j}^{n}-2 u_{i, j}^{n}\right)+(\Delta y)^{-2}\left(u_{i, j+1}^{n}+u_{i, j-1}^{n}-2 u_{i, j}^{n}\right) \\
& \because \bar{L}\left(u_{i, j}^{n}\right)=a(\Delta x)^{-2}\left(u_{i+1, j}^{n}+u_{i-1, j}^{n}-2 u_{i, j}^{n}\right)+c(\Delta y)^{-2}\left(u_{i, j+1}^{n}+u_{i, j-1}^{n}-2 u_{i, j}^{n}\right)+ \\
& \quad b(2 \Delta x \Delta y)^{-1}\left(u_{i+1, j+1}^{n}+u_{i-1, j-1}^{n}-u_{i+1, j-1}^{n}-u_{i-1, j+1}^{n}\right)
\end{aligned}
$$

The convergence rate of Eq. (2.2) will be" analyzed assuming

$$
e_{i, j}^{n} \equiv u_{i, j}^{n}-u_{i, j}=e^{n} \exp \left[I\left(i k_{x} \Delta x+j k_{y} \Delta y\right)\right], \cdots \quad I \equiv \sqrt{-1}
$$

where $u_{j} j$ is the converged solution, $e^{n}$ the error amplitude and $\vec{k} \equiv\left(k_{x}, k_{y}\right)$ the propagation vector. Assuming that $\vec{k} \neq 0$ and using the following defintions: 


$$
\begin{gathered}
{ }_{x} \equiv\left(k_{x} \Delta x\right) / 2, a_{x} \equiv\left(\sin \theta_{x}\right) / \Delta x, \theta_{y} \equiv\left(k_{y} \Delta y\right) / 2, a_{y} \equiv\left(\sin \theta_{y}\right) / \Delta y \\
s_{x} \equiv a_{x} /\left[\left(a_{x}\right)^{2}+\left(a_{y}\right)^{2}\right]^{1 / 2}, s_{y} \equiv a_{y} /\left[\left(a_{x}\right)^{2}+\left(a_{y}\right)^{2}\right]^{1 / 2}
\end{gathered}
$$

then Eqs. (2.2) and (2.5) imply that

$$
e^{n+1} / e^{n}=E(\tau, \vec{k}) \equiv 1-\tau G(\vec{k}) \quad(\vec{k} \neq 0)
$$

with $\quad \dot{G}(\vec{k}) \equiv a\left(s_{x}\right)^{2}+c\left(s_{y}\right)^{2}+2 b s_{x} s_{y} \cos \theta_{x} \cos \theta_{y}$

It should be noted that the von Neumann analysis (along with the condition $\vec{k} \neq 0$ ) can be fully justified if it is applied to a grid with periodic boundary conditions.

Let $\sigma_{\max }$ and $\sigma_{\min }$, respect ively, be the greatest and the smallest eigen-

values of the symmetric and positive-definite matrix $\left(\omega_{0}, v=1,2\right)$

$$
A \equiv\left(\alpha_{\mu v}\right), \quad \alpha_{11}=a, \alpha_{12}=a_{21}=b, \alpha_{22}=c
$$

Then it can be shown that 5

$$
\sigma_{\max } \geq G(\vec{k}) \geq \sigma_{\min }>0
$$

with the understanding that the bounds $\sigma_{\max }$ and $\sigma_{\min }$ are sharp if all the allowable $\vec{k}(\neq 0)$ are considered. As a result, the asymptotic error amplification factor $E(\tau)(\equiv$ the supremum of $|E(\tau, \vec{k})|$ for a given $\tau$ ) will reach its minimum

$$
E_{0} \equiv E\left(r_{0}\right)=(\Sigma-1) /(\Sigma+1)<1
$$

when $t=\tau_{0} \equiv$ the optimal relaxation factor $=2 /\left(\sigma_{\max }+\sigma_{\min }\right)=2 /(a+c)$.

Here $\Sigma$ is the condition number $\equiv \sigma_{\max } / \sigma_{\min }>1$.

Equations (2.1)-(2.13) can be easily generalized for $\mathrm{N}$-dimensional problems: The only exception is Eq. (2.13) where the last equality sign is not valid if $N>2$.

\section{LOCAL RELAXATION}

The variable coefficient (VC) version of the numerical procedure presented in Section 2 is obtained by replacing the constant coefficients $a, b, c, T, \tau_{0}$, $\sigma_{\max }$, and $o_{\text {min }}$ in Eqs. (2.2) to (2.4) and (2.13), respectively, with the grid point dependent coefficients $a_{i j}, b_{i j}, c_{i j}, \tau_{i j}, \tau_{0}, i j, \sigma_{\max }, j_{j}$ and $\sigma_{m i n, i j}$ : Obviously, the VC version is well defined at arl interinr grid points. For a grid point on a periodic or Neumann boundary, it can also be defined using the periodic condition or an extrapolation technique explained in Ref. 6 .

The current procedure $c$ an be modified to solve PDE with

$$
L=L^{\prime}(x, y) \equiv \frac{\partial}{\partial x}\left(p(x, y) \frac{\partial}{\partial x}\right)+\frac{\partial}{\partial y}\left(q(x, y) \frac{\partial}{\partial y}\right)
$$

where $p$ and $q$ are arbitrary positive functions of $x$ and $y$. With $L^{\prime}=L^{\prime}(x, y)$, the VC version of Eq. (2.2) can be obtained by replacing $\left[\left(u_{1}^{0}, j\right)\right.$ with ${ }^{7}$

$$
\begin{gathered}
\check{L}^{\prime}\left(u_{i, j}^{n}\right) \equiv(\Delta x)^{-2}\left[p_{(i-1 / 2) j} u_{i-1, j}^{n} p_{(i+1 / 2) j} u_{j+1, j}^{n}-\left(p_{(i-1 / 2) j}{ }^{+p}(i+1 / 2) j\right.\right. \\
\left.u_{i, j}^{n}\right]+ \\
(\Delta y)^{-2}\left[q_{i(j-1 / 2)} u_{i, j-1}^{n}+q_{i(j+1 / 2)} u_{i, j+1}^{n}-\left(q_{i(j-1 / 2)} q_{i(j+1 / 2)}\right) u_{i, j}^{n}\right]
\end{gathered}
$$

In case that the values of $p$ and $q$ do not vary greatly from one grid point to its neighbors, then $\tilde{L} \cdot\left(u_{i, j}^{n}\right) \div \tilde{L}\left(u_{i, j}^{n}\right)$ assuming $a_{i j}=p_{i j}, c_{i j}=q_{i j}$ and $b_{i j}=0$. This observation coupled with Eq. (2.13) leads us to the assumption:

$$
\tau_{i j}=\tau_{0, i, j}^{\prime} \equiv 2 /\left(p_{i j}+q_{i j}\right) \quad\left(L=L^{\prime}(x, y)\right)
$$




\section{SCALING}

Assuming that the definition of the operator $P$ is broadened as

$$
\vec{P}\left(u_{i, j}^{n}\right)=g_{x}(\Delta x)^{-2}\left(u_{i+1, j}^{n}+u_{i-1, j}^{n}-2 u_{i, j}^{n}\right)+g_{y}(\Delta y)^{-2}\left(u_{i, j+1}^{n}+u_{i, j-1}^{n}-2 u_{i, j}^{n}\right)
$$

with $g_{x}$ and $g_{y}$ positive constants, then the only modifications required for Sec: 2 are to replace the coefficients $a_{x}, a_{y}, a, b$, and $c$ in Eqs. (2.7), (2.9), (2.10) and (2.13), respectively, with $\sqrt{g_{x}} a_{x}, \sqrt{g_{y} a_{y}}, a / g_{x}, b / \sqrt{g_{x} g_{y}}$ and $c / g_{y}$. Since $g_{x}$ and $g_{y}$ are positive constants, their appearance in Eq. $(4.1)$ does not -increase the difficulty of inverting $\widetilde{p}$. However, their introduction into the current iterative procedure does have an effect on the convergence rate. As a result, the current iterative procedure can be accelerated considerably by a proper choice of $g_{x}$ and $g_{y}$. Obviously, this scaling technique can also be used in the solution of Pó with variable coefficients as long as the scaling coefficients $g_{x}$ and $g_{y}$ remain positive constants.

\section{NUMERICAL EVALUATION}

To facilitate this discussion, we begin with the following preliminaries:

(a) the residual norm $r(n)$ and error norm $e(n)$ after $n$ iterations are defined by:

$$
r(n) \equiv\left[\sum_{i, j}\left(\tilde{L}\left(u_{i, j}^{n}\right)-h_{i j}\right)^{2}\right]^{1 / 2} \text { and } e(n) \equiv\left[\sum_{i, j}\left(u_{i, j}^{n}-u_{i, j}^{\infty}\right)^{2}\right]^{1 / 2}
$$

where $u_{i, j}^{\infty}$ is the machine accuracy solution. Moreover, we def ine

$$
0_{r}(n) \equiv-\log _{10}[r(n) / r(0)] \text { and } 0_{e}(n) \equiv-\log _{10}[e(n) / e(0)]
$$

(b) We assume that $u_{i, j}^{0}=0$ at all grid points where $u_{j, j}$ 's are unknowns." As a result, e(o) ${ }^{i}\left\|_{11}^{\infty}\right\|$ and $0_{e}(n)$ is the number of correct digits in $u_{i, j}^{n}$

(c) The coefficient. $E_{0, j j}$ defined by the VC version of Eq. (2.12) may be interpreted as an asymptotic error amplification factor. As a result, the value of $0_{e}(n)$ (and $0_{r}(n)$ if $\left.0_{r}(n) \sim 0_{e}(n)\right)$ may be predicted by the parameter

$$
0_{t}(n) \equiv-n \log _{10} \text { (the supremum of all } E_{0, i j} \text { ) }
$$

where $E_{0, i j}$ is evaluated assuming $a_{i j}=p_{i j}, b_{i j}=0$ and $c_{i j}=q_{i j}$ if $L=L^{\prime}(x, y)$. Alternatively, the value of $0_{e}(n)$ or $0_{r}(n)$ may be predicted by $\overline{0}_{t}(n) \equiv$ the limit of $0_{t}(n)$ as the grid cell size approaches zero.

(d) The domain for all numerical problems is assumed to be $1 \geq x \geq 0$ and $1 \geq y \geq 0$. Moreover, the iterative procedure is executed using aan FDE code ${ }^{8}$ with $g_{x}=g_{y}=1$.

The first group of PDE to be studied in this section includes:

$$
\begin{aligned}
& \left(1+2 x^{2}+2 y^{2}\right) \partial^{2} u / \partial x^{2}+\left(1+x^{2}+y^{2}\right) \partial^{2} u / \partial y^{2}=1 \\
& \left(1+2 x^{2}+2 y^{2}\right) \partial^{2} u / \partial x^{2}+\left(1+x^{2}+y^{2}\right) \partial^{2} u / \partial x \partial y+\left(1+x^{2}+y^{2}\right) \partial^{2} u / \partial y^{2}=1 \\
& \frac{\partial}{\partial x}\left[\left(1+\left(x^{2}+y^{2}\right)^{\ell}\right\} \frac{\partial u}{\partial x}\right]+\frac{\partial}{\partial y}\left[\left(2+\left(x^{2}+y^{2}\right)^{\ell}\right) \frac{\partial u}{\partial y}\right]=1, \ell=2,4,6,8
\end{aligned}
$$

Ten numerical problems associated with Eqs. (5.4)-(5.6) are defined in Table 1 . The parameters $M X$ and MY, respectively, are the number of grid intervals in the $x$ and $y$ directions. It is assumed that $u=0$ at all boundaries.

:Problems 1-3 are associated with Eq. $(5.4)$. They differ only in the grid cell size and aspect ratio. As shown in Table 1; for all three problems, the value of $0_{t}(20)$ or $\overline{0}_{t}(20)$ provides a conservative estimate of $0_{r}(20)$ (since $0_{e}(n) \sim 0_{r}(n)$, the values of $0 \mathrm{e}(n)$ are not listed). It is also seen that the very large aspect ratio (16:1) of the grid cell in problem 3 does not cause any substantial reduction in convergence rate. Problems 4-6 represent a parallel study for Eq. $(5.5)$. It is 
evident that the appearance of a cross derivative term in Eq. (5.5) causes an increase in the conservatism of the theoretical est imate made by $0_{t}(n)$ or $\overline{0}_{t}(n)$ : This is particularly true if the grid cell size or aspect ratio is large. For problems 1-6, the convergence $h$ istories are closely represented by straight 1 ines (as an example, see Fig. 1) as would be expected from our theoretical study.

Equations (5.6) belong to the class of PDE defined by Eq. (3.1). It is evident that the variation of the coefficients $p$ and $q$ increases progressively as one goes from $l=2$ to $l=4$ and so on. For $l=8$, the increase in the values of $p$ and $q$ from one corner $(x=y=0)$ to another corner $(x=y=1)$ on the unit square is of the order of 100 times. As shown in Fin. 2, the current method is still useful. in this extreme case. The robustness of this algorithm is most evident in its ability to reverse the trend toward divergence during the first few iterations. The second group of PDE to be studied includes

$$
\begin{aligned}
& \frac{\partial}{\partial x}\left[\left(1+(x+y)^{2}, \frac{2 \partial u}{\partial x}\right]+\frac{\partial}{\partial y}\left[\left(1+\sin ^{2}(x+y)\right)^{2} \frac{\partial u}{\partial y}\right]=h_{1}(x, y)\right. \\
& \frac{\partial}{\partial x}\left[\left(1+\frac{1}{2}\left(x^{4}+y^{4}\right)\right\}^{2} \frac{\partial u}{\partial x}\right]+\frac{\partial}{\partial y}\left[\left(1+\frac{1}{2}\left(x^{4}+y^{4}\right)\right)^{2} \frac{\partial u}{\partial y}\right]=h_{2}(x, y)
\end{aligned}
$$

Here $h_{1}(x, y)$ and $h_{2}(x, y)$ along with the boundary values of $u$ are chosen such that

$$
u=u_{1}(x, y) \equiv \sin x \text { siny and } u=u_{2}(x, y) \equiv[x(1-x) y(1-y)]^{2}
$$

respectively, are the exact solutions of Eq. (5.7) and (5.8). Both Eqs. (5.7) and (5.8) are solved numerically assuming $M X=M Y=16$. Both numerical problems are test problems used by Bank ${ }^{4}$. Compared with the current value of $0_{e}(10)=3.47$, the values obtained by Bank for Eq. (5.7) are 3.49 without using his scaling technique and $3.87,4.81$, and 6.76 using different scaling functions. Since the operator used by Bank is a general separable operator, the computation time required for its inversion is about six times that required for the current Poisson-like operator ( $p$. 5 of ref. 1). As a result, the current method is at least a factor of 2 more efficient even without using our scaling technique. For Eq. (5.8), compared with the current value of $0_{\mathrm{e}}(10)=8.59$, the values reported by Bank are 5.88 without using scaling technique and 14.79 if a scaling function is used. Equation (5.8) was also solved by Concus and Golub ${ }^{3}$ and the results are comparable with ours. However their method is applicable only when $p(x, y)=q(x, y)$ as in the case of Eq. (5.8).

\section{APPLICATION TO A 3-D INTERNAL FLOW PROBLEM}

In a numerical study. (to be published) of 3-D incompressible shear flow in a turning channel (Fig. 3), the elliptic PDE

$$
\partial^{2} u / \partial x^{2}+\partial^{2} u / \partial y^{2}+n(x, y) \partial^{2} u / \partial z^{2}=h,(2 \geq x \geq-2,0.75 \geq y \geq 0.65,0.1 \geq z \geq 0)
$$

is solved in computational space. Here $h$ is a known source term and

$$
n(x, y)=(\cosh (\pi x)+\cos (\pi y)) /(\cosh (\pi x)-\cos (\pi y))>0
$$

It is assumed that $u=0$ at $x=2$ and the normal derivative of $u$ vanishes at other boundaries. Since Eq. (6.1) has a form specified by Eq. (1.1) and the 3-D version of Eq. (2.1), it was solved numerically using the current iterative method with a.144 $\times 12 \times 12$ uniform grid. To accelerate the convergence, the scaling coefficients of the 3-D operator $P$ (see Eq. (4.1)) are given by

$$
\mathrm{g}_{\mathrm{x}}=\mathrm{g}_{\mathrm{y}}=1, \mathrm{~g}_{3}=\sqrt{\eta_{\max } \cdot \eta_{\max }} \div 0.4135
$$

where $\eta_{\max }(=0.9966)$ and $\eta_{\min }(=0.1716)$, respectively, are the max imum and the minimum of $n$ in the domain of Eq. (6.1). It should be noted that the maximum value of $\overline{0}_{t}(1)(=0.415)$ is reached if the values of $g_{x}, g_{y}$, and $g_{z}$ are chosen

according to Eq. $(6.3)$. 
Equation (6.1) was numerically solved many times with different source terms $h$. Without exception, it was found that, after four iterations, the slope of $0_{r}(n)$ vs $n$ curve is predicted by the value of $\overline{0}_{t}(1)$ to within ten percent.

\section{REFERENCES}

1. Hockney, R. H.: "Rapid Elliptic Solvers," Numerical Mèthods in Applied Fluid Dynamics edited by B. Hunt, Academic Press, 1-48 (1980).

2. D'yakonove, E. G.: Dok 1. Akad, Nauk, SSSR, 138, 522-525.(1961).

3. Concus, P. and Golub, 6. H.: SIAM J. Numer. Anal., 10, 1103-1120 (1973).

4. Bank, R. E.: SIAM J. Numer. Añal., 14, 950-970 (1977).

5. Chang, S. C.: "Generalizations of Two Inequalities Involving Hermitian Forms," - accepted for publication in Linear Algebra and Its Applications.

6. Smith, G. D.: "Numerical SoTution of Partial Differential Equations," 0xford University Press. p. 29 (1978).

7. Hageman, L. A. and Young, D. M.: "Applied Iterative Methods," Academic Press, p. 12 (1981).

8. Adams, J., Swartztrauber, P. and Sweet, R.: "FISHPAK: A Package of FORTRAN Subprograms for the Solution of Separable Elliptic Partial Differential Equations, Version 3," National Center for Atmospheric Research, Boulder, Colorado, June 1979. 
Table 1. ( $n=20$ for Probs. 1-3, $n=32$ for Probs. 4-10)

\begin{tabular}{|c|c|c|c|c|c|c|c|}
\hline $\begin{array}{c}\text { Prob. } \\
\text { No. }\end{array}$ & Eq. No. & $\&$ & $M X$ & $M Y$ & $0_{r}(n)$ & $0_{t}(n)$ & $0_{t}(n)$ \\
\hline 1 & $(5.4)$ & NA & 16 & 16 & 14.5 & 12.3 & 12.0 \\
2 & $(5.4)$ & NA & 64 & 64 & 13.8 & 12.1 & 12.0 \\
3 & $(5.4)$ & NA & 64 & 4 & 14.0 & 12.7 & 12.0 \\
4 & $(5.5)$ & NA & 16 & 16 & 14.4 & 9.7 & 9.6 \\
5 & $5.5)$ & NA & 64 & 64 & 12.6 & 9.6 & 9.6 \\
6 & $(5.5)$ & NA & 64 & 4 & 19.0 & 10.0 & 9.6 \\
7 & $5.6)$ & 2 & 16 & 16 & 17.2 & 15.3 & 15.3 \\
8 & $(5.6)$ & 4 & 16 & 16 & 16.8 & 15.3 & 15.3 \\
9 & $(5.6)$ & 6 & 16 & 16 & 13.4 & 15.3 & 15.3 \\
10 & $(5.6)$ & 8 & 16 & 16 & 7.6 & 15.3 & 15.3 \\
\hline
\end{tabular}




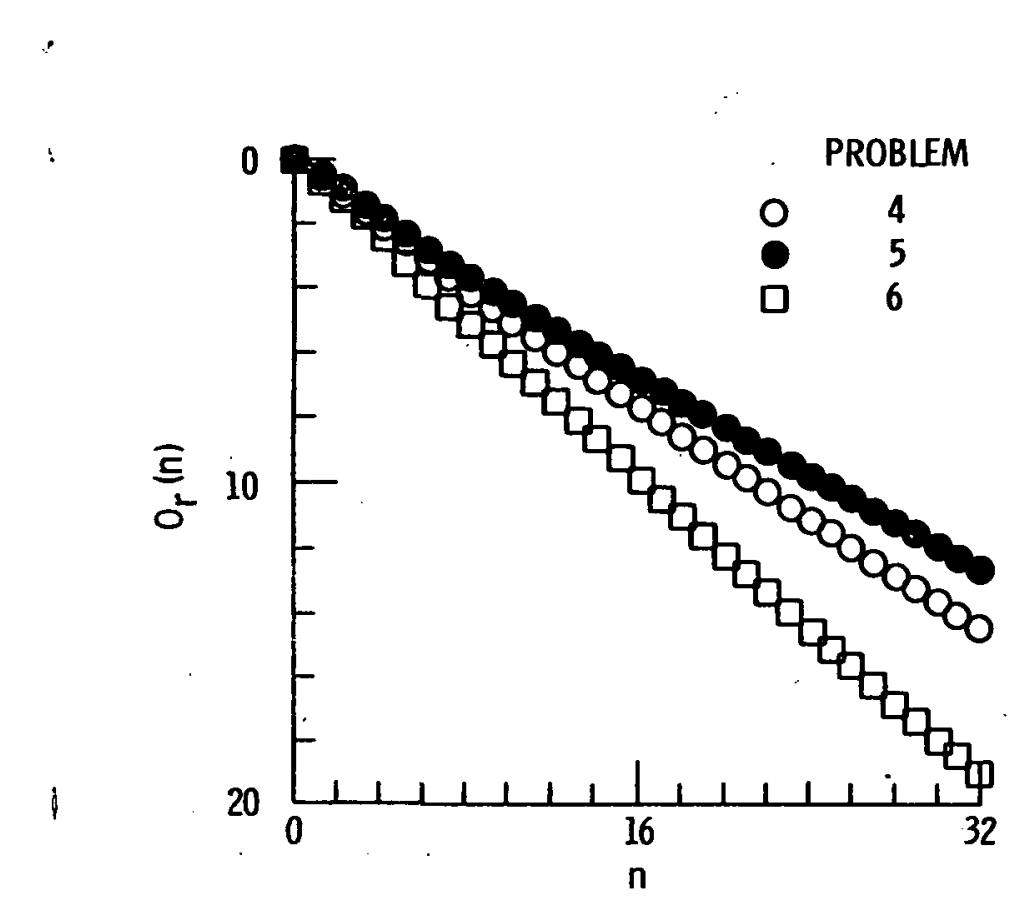

Figure 1. - Convergence histories of Problems 4-6.

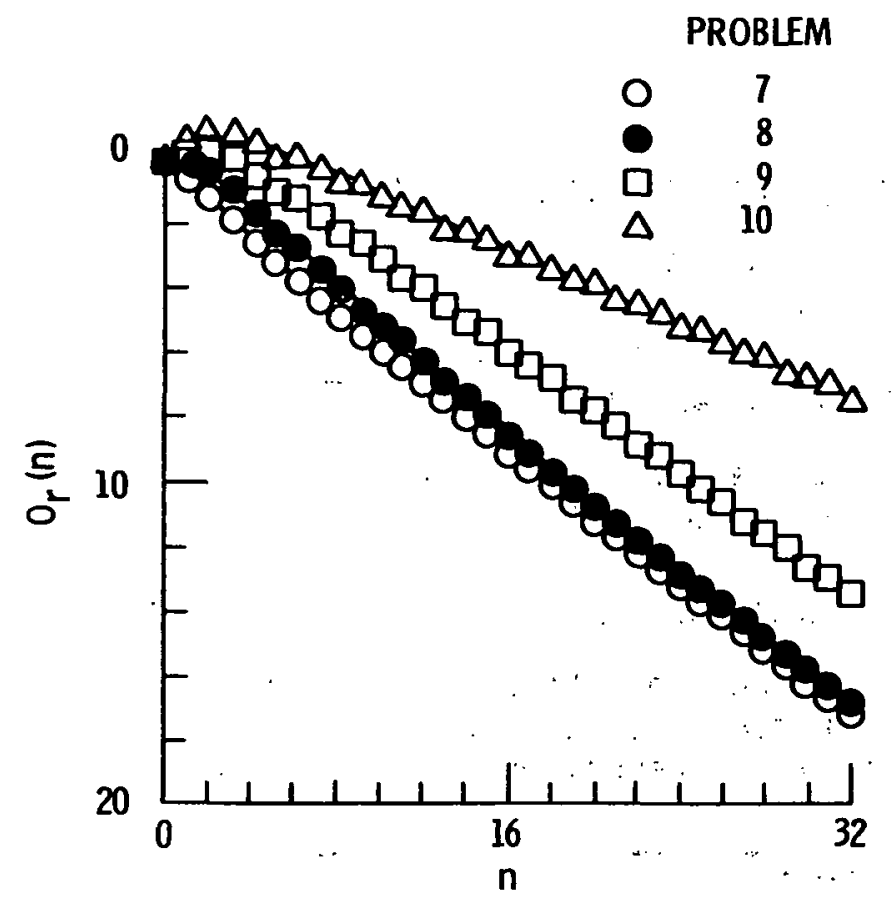

Figure 2 - Convergence histories of Problems 7-10.

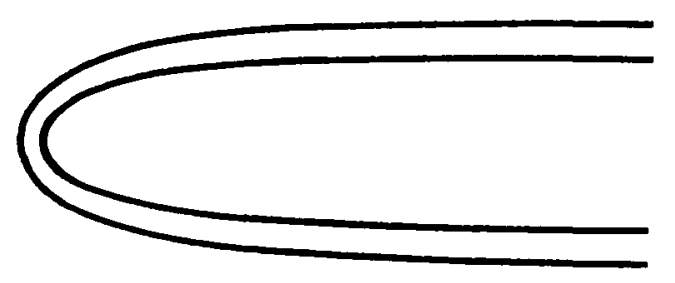

Figure 3. - A converging-diverging turning channel in physical space. 


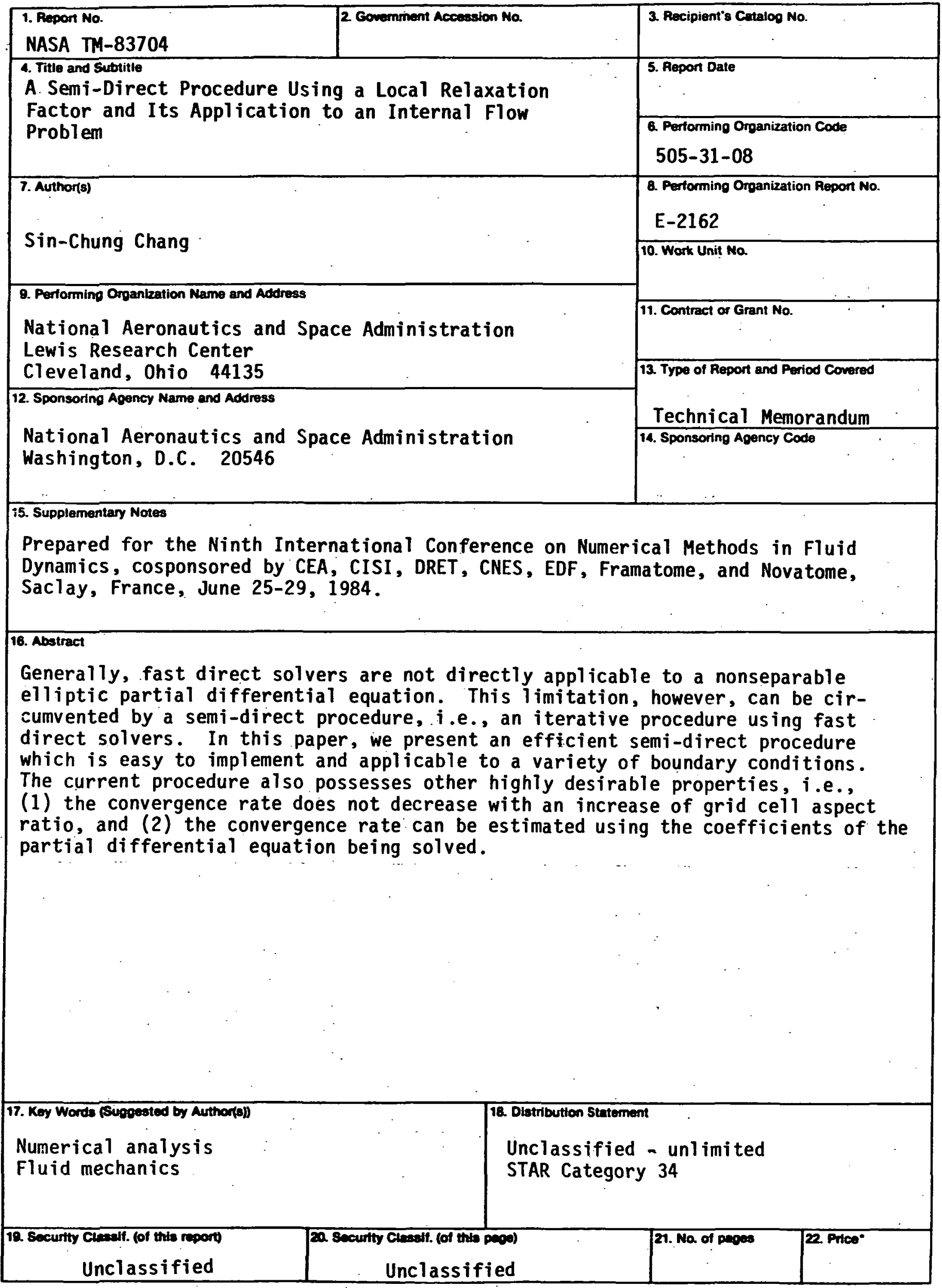

'For sale by the National Technical Information Service, Springfield. Virginia 22161 
National Aeronautics and Space Administration

Washington, D.C.

20546

Official Business

Penalty for Private Use, $\mathbf{5 3 0 0}$
SPECIAL FOUATH CLASS MAIL cook

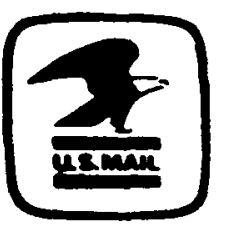

Poxtege and Fees Paid Netional Aeronautics and Spece Adminiztration NASA-451 